\title{
PARALLEL BEAM DYNAMICS SIMULATION TOOLS FOR FUTURE LIGHT SOURCE LINAC MODELING*
}

\begin{abstract}
Ji Qiang, Ilya v. Pogorelov, Robert D.
Abstract

Large-scale modeling on parallel computers is playing an increasingly important role in the design of future light sources. Such modeling provides a means to accurately and efficiently explore issues such as limits to beam brightness, emittance preservation, the growth of instabilities, etc. Recently the IMPACT codes suite was enhanced to be applicable to future light source design. Simulations with IMPACT-Z were performed using up to one billion simulation particles for the main linac of a future light source to study the microbunching instability. Combined with the time domain code IMPACT-T, it is now possible to perform large-scale start-to-end linac simulations for future light sources, including the injector, main linac, chicanes, and transfer lines. In this paper we provide an overview of the IMPACT code suite, its key capabilities, and recent enhancements pertinent to accelerator modeling for future linac-based light sources.
\end{abstract}

\section{INTRODUCTION}

Next generation linac-based free electron laser (FEL) light sources present great opportunities for scientific discovery and applications. A number of FEL projects are under construction and are being proposed. To design these light sources, many technical challenges must be overcome. One example involves predicting and controlling the growth of the microbunching instability driven by longitudinal space charge (LSC). Another example, Coherent Synchrotron Radiation (CSR), is particularly important because it can lead to irreversible degradation in beam quality, and ultimately reduce FEL output performance.

Large-scale simulation on parallel computers has two main benefits: First, it can be used to perform smalland medium-scale simulations much more quickly than serial computer simulations. Second, large-scale simulation makes it possible to perform simulations with greater resolution and accuracy (lower numerical shot noise) than serial simulations, so much so that in many instances serial simulations would be impossible to perform. This is especially important in order to accurately predict the final beam quality in the presence of the microbunching instability.

The IMPACT code suite consists of two parallel particlein-cell tracking codes (one is longitudinal position dependent and one is time dependent), an rf linac lattice design code, an envelope matching and analysis code, and a number of pre- and post-processing codes. Both parallel particle tracking codes assume a quasi-static model of the beam

\footnotetext{
* Work supported by the Office of Science, U. S. Department of Energy, under Contract No. DE-AC02-05CH11231

† rdryne@lbl.gov
}

and calculate space-charge effects self-consistently at each time step together with the external acceleration and focusing fields. The IMPACT code suite has been used to study the beam dynamics in a number of projects $[1,2,3,4,5]$.

\section{MODELING ELECTRON BEAM DYNAMICS IN PHOTOINJECTORS}

Electrons generated from a photocathode are modeled using a time-dependent parallel particle-in-cell code, IMPACT-T, in photoinjectors [6]. Using time as the independent variable in this regime is necessary due to the fact that the space-charge interaction, in the quasi-static approximation, occurs at a given time and is a dominant factor affecting electron beam dynamics in photoinjectors. Some recent improvements to the IMPACT-T code include: short range structure wakefield effects using an analytical wake function or read in data file, a flag to switch on/off photocathode effect, a new 1D CSR wake module including transient effects at the entrance and the exit of a dipole bend magnet, inclusion of field variation inside the fringe field region of magnets using an Enge function, and a fringe field option for quadrupole elements. A detailed description of the IMPACT-T code can be found in reference [7]. Fig. 1

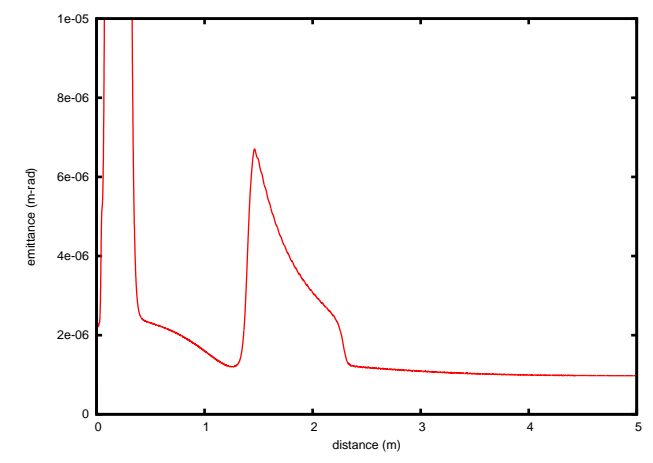

Figure 1: Transverse emittance evolution in a photoinjector.

shows an example of transverse projected emittance evolution in a photoinjector similar to the LCLS design with the presence of both pi mode and 0 mode. Here, the 0 mode frequency is $3.5 \mathrm{MHz}$ lower than the pi mode with a maximum amplitude of $12 \mathrm{MV} / \mathrm{m}$ with the same phase.

\section{SIMULATION OF ELECTRON BEAM TRANSPORT IN LINACS}

Electron transport through a linac is simulated using a position-dependent parallel particle-in-cell code, IMPACT$\mathrm{Z}$ [8]. Using position as the independent variable takes ad- 
vantage of the position dependent modeling of external elements and speeds up the computation by using transfer maps. The space-charge effects in this regime are small, and the commonly used ballistic approximation to go from a position dependent distribution to a time dependent distribution is valid. To model the electron beam in an $\mathrm{rf}$ linac we have added new capabilities to the code. These capabilities include: modeling forward and backward traveling wave rf structures, modeling 3D space-charge effects using an integrated Green function solver from the IMPACT-T code, modeling short range wake fields, and modeling coherent synchrotron radiation effects in bending magnets.

For a beam out of the injector, the electrons move inside the linac with a speed close to the speed of light. The relative longitudinal motion among electrons is small except inside the chicane. This fact can be used to speed up the simulation by lumping the space-charge and wake field calculation in most sections of the linac. Inside the chicane, where the relative motion is strong, multiple space-charge and CSR wakefield kicks are used. By doing the spacecharge/wakefield lumping, we can save the computational time by a factor of four to ten. However, this results in some loss of information in the transverse plane. Fig. 2 shows a

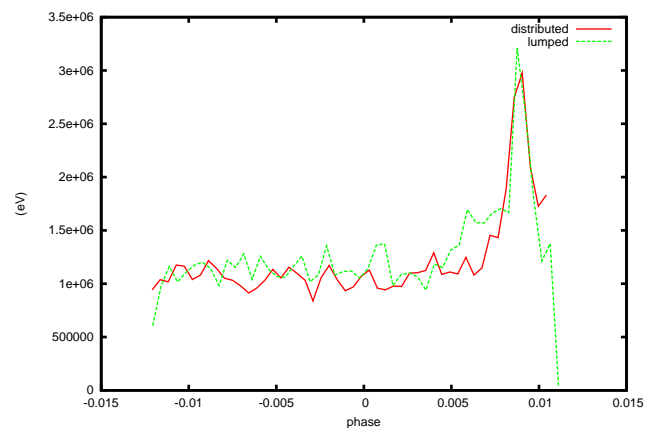

Figure 2: Uncorrelated energy spread at the end of a linac using distributed and lumped space charge/wakefield kicks.

comparison of the final sliced rms energy spread from the lumped simulation and the standard distributed simulation. The level of energy spread shows reasonably good agreement between the two simulations.

The IMPACT code was developed as a parallel particlein-cell code using a domain decomposition method. In this method, the spatial computational domain is divided and assigned to individual processors. Macroparticles with physical position in that computational domain are assigned to that processor. During each time step, when a particle moves out of the original computational domain, it will be sent to the other processor owning that spatial domain containing the particle's new position. This method has the advantage of avoiding global communication during the charge deposition to obtain the charge density distribution on the grid and during the field interpolation to obtain the space-charge fields from the grid. Only neighboring communication is needed in these stages and in the particle moving stage. However, this method may suffer from unbalanced work since each processor may have a different number of macroparticles for a non-uniform particle distribution. In order to maintain a roughly equal number of particles on each processor, the local computational domain boundary of each processor has to adjusted frequently. This introduces extra computational cost and may not even work well for some types of distributions. To overcome this problem, we also implemented another parallel algorithm, a particle-field decomposition method, into the IMPACT code. This algorithm is based on a uniform distribution of macroparticles and computational domain among individual processors, and is a technique which has been used in our previous simulation of colliding beams [9]. In this algorithm, there is no particle moving among processors and each processor keeps about the same amount of work. However, during the charge-deposition and the field interpolation stages, the information has to be exchanged globally among all processors. For some applications, this could present a significant slow down of the code. In the simulation of beam transport through a linac for light source design, a large number of macroparticles (e.g. one billion) is used to reduce numerical shot noise in order to accurately predict the microbunching instability. In this case, the number of computational grid points may be much less than the number of macroparticles. Using a particle-field decomposition method helps to reduce the usage of memory on a single processor by keeping a good load balance and also improves the computational speed.

With these recent enhancements, we have used the IMPACT code to simulate electron beam transport through the designed FEL linac for the Fermi@Elettra project at Trieste. In these simulations, we have used an initial distribution out of the injector generated by Fermi researchers. This particle distribution contains only 200,000 particles. We have repopulated this particle distribution by resampling a transverse four dimensional uniform distribution centered one each original particle. The size of the uniform distribution in each dimension is chosen to smooth the distribution and to keep the rms size and emittance close to the original distribution. In the longitudinal phase space, we cut the distribution into a number of slices along the $\mathrm{z}$ direction. Within each slice, we use a linear function to fit the particle distribution. Each particle is resampled in longitudinal phase space centered on the original particle position. The energy deviation is calculated from the fitted correlated energy distribution plus a uniform sampling with desired initial energy spread to emulate the effects of a laser heater. Fig. 3 shows the uncorrelated rms energy spread at the end of the linac with a different number of slices and one billion macroparticles. Here, the larger slice number corresponds to a smaller longitudinal box size and less initial smoothing. It can be seen that when the initial box size is chosen too small (2000 slices), there is significant energy spread growth of numerical noise due to the microbunching instability even using one billion macroparticles. As the initial box size gets larger, the initial numerical shot noise is significantly reduced by using one billion particles. Be- 


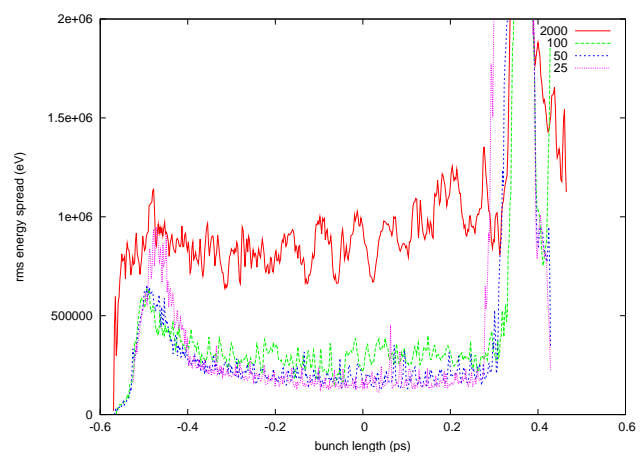

Figure 3: Uncorrelated energy spread at the end of the linac with initial $15 \mathrm{keV}$ energy spread and initial longitudinal resampling using 25, 50, 100 and 2000 slices.

tween 25 and 50 slices there is a convergence of the final energy spread with respect to the initial box size.

Given an initial repopulation box size, the number of macroparticles used in the simulations affects the final energy spread growth through the numerical shot noise. For a real electron beam with five billion electrons, we would like to use a large number of macroparticles in order to accurately predict the energy spread growth driven by the microbunching instability. Fig. 4 shows a longitudinal phase space plot at the end of the linac simulated using 10 million and 1 billion macroparticles. It is seen that using 10 million

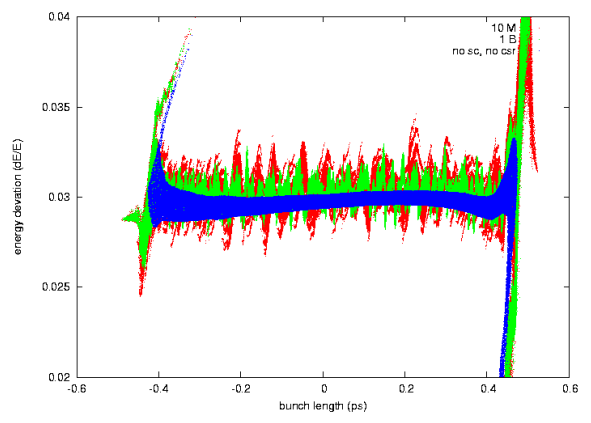

Figure 4: Longitudinal phase space distribution at the end of the linac from simulations using 10 million and 1 billion macroparticles together with the case without space-charge and CSR effects.

macroparticles is not sufficient to accurately predict the final energy spread growth.

The initial uncorrelated energy spread provides a way to decohere the particle motion and reduce the growth of the microbunching instability. A smaller initial energy spread will have less effect on the microbunching instability and hence larger final growth of energy spread. Fig. 5 shows the uncorrelated energy spread at the end of the linac using initial $7.5 \mathrm{keV}$ and $15.0 \mathrm{keV}$ uncorrelated energy spread. As expected, $7.5 \mathrm{keV}$ initial uncorrelated energy spread has a larger final energy spread at the end of the linac than the

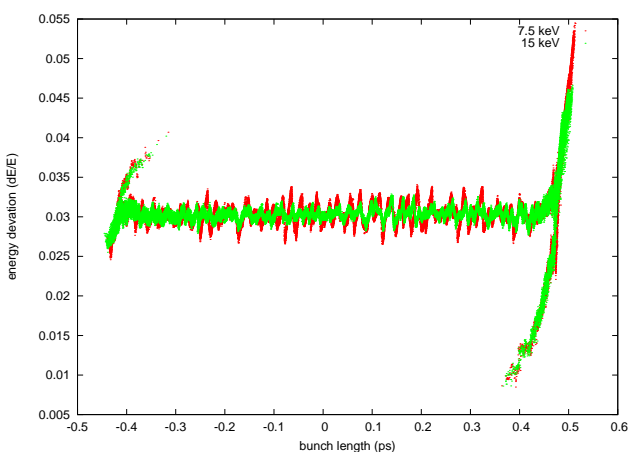

Figure 5: Longitudinal phase space distribution at the end of the linac from a simulation using 1 billion macroparticles with initial $7.5 \mathrm{keV}$ and $15 \mathrm{keV}$ uncorrelated energy spread.

$15 \mathrm{keV}$ initial energy spread.

\section{ACKNOWLEDGMENTS}

This research used resources of the National Energy Research Scientific Computing Center, which is supported by the Office of Science of the U.S. Department of Energy under Contract No. DE-AC02-05CH11231. We thank our Trieste collaborators on the Fermi@Elettra project for helpful discussions and for providing us with the initial particle distributions and linac lattice used in these studies. We thank our LBNL collaborators, J. Corlett, W. Fawley, M. Venturini, and S. Zholents, for helpful discussions.

\section{REFERENCES}

[1] J. Qiang, et al, Nuclear Instruments \& Methods in Physics Research A457, 1, (2001).

[2] M. Ikegami, et al, "Comparison of Particle Simulation with J-PARC Linac MEBT Beam Test Results," Proc. HALO03, May 19-23, Montauk, NY, p. 96, (2003).

[3] F. Gerigk, M. Vretenar, R. D. Ryne, "Design of the superconducting section of the SPL linac at CERN," Proc. PAC01, p. 3909, (2001).

[4] J. Qiang, P. L. Colestock, D. Gilpatrick, H. V. Smith, T. P. Wangler, and M. E. Schulze, Phys. Rev. ST Accel. Beams, Vol 5, 124201 (2002).

[5] R. Garnett, et al, "Advanced Beam-Dynamics Simulation Tools for RIA,” Proc. PAC2005, Knoxville, Tennessee, May 16-20, p. 4218, (2005).

[6] J. Qiang, S. Lidia, R. D. Ryne, C. Limborg-Deprey, Phys. Rev. Special Topics - Accel. Beams 9, 044204, (2006).

[7] J. Qiang, "IMPACT-T User Document Version 1.5", LBNL rep 62326, 2007.

[8] J. Qiang, R. Ryne, S. Habib, and V. Decyk, J. Comp. Phys. 163,434 (2000).

[9] J. Qiang, M. Furman, and R. Ryne, J. Comp. Phys. vol. 198, 278 (2004). 